\title{
Quantum Theory of Cavity-Assisted Sideband Cooling of Mechanical Motion
}

\author{
Florian Marquardt, ${ }^{1}$ Joe P. Chen, ${ }^{2,4}$ A. A. Clerk, ${ }^{3}$ and S. M. Girvin ${ }^{2}$ \\ ${ }^{1}$ Department of Physics, Arnold-Sommerfeld-Center for Theoretical Physics, and Center for NanoScience, \\ Ludwig-Maximilians-Universität München, Theresienstrasse 37, 80333 Munich, Germany \\ ${ }^{2}$ Department of Physics, Yale University, P.O. Box 208120, New Haven, Connecticut 06520-8120, USA \\ ${ }^{3}$ Department of Physics, McGill University, 3600 rue University, Montreal, QC Canada H3A $2 T 8$ \\ ${ }^{4}$ Department of Physics, Cornell University, 109 Clark Hall, Ithaca, New York 14853-2501, USA
}

(Received 22 January 2007; published 28 August 2007)

\begin{abstract}
We present a quantum-mechanical theory of the cooling of a cantilever coupled via radiation pressure to an illuminated optical cavity. Applying the quantum noise approach to the fluctuations of the radiation pressure force, we derive the optomechanical cooling rate and the minimum achievable phonon number. We find that reaching the quantum limit of arbitrarily small phonon numbers requires going into the goodcavity (resolved phonon sideband) regime where the cavity linewidth is much smaller than the mechanical frequency and the corresponding cavity detuning. This is in contrast to the common assumption that the mechanical frequency and the cavity detuning should be comparable to the cavity damping.
\end{abstract}

Recent years have seen significant advances in fabricating, measuring, and controlling mechanical systems on the micro- and nanometer scale [1-4]. A number of experiments to explore the quantum regime of these mechanical devices have been proposed, including generation of nonclassical states [5], entanglement [6-11], and quantumlimited measurements [12,13]. Many of these proposals require the system to be cooled to its ground state, i.e., to temperatures below $20 \mathrm{mK}$ even for $1 \mathrm{GHz}$ resonators. This is hard or impossible using bulk refrigeration, but it may be feasible using nonequilibrium cooling techniques analogous to the laser-cooling schemes for trapped ions and neutral atoms [14].

Cooling via an active feedback loop [15-17] has already been considered elsewhere (including its quantum limits [18]). Here we will focus on passive cooling (without feedback). In this approach the cantilever displacement is coupled parametrically to a driven resonator (or two-level system). When the drive frequency is chosen appropriately, the cantilever is cooled. The lowest achievable temperature is determined by the resonator's quantum fluctuations (photon shot noise).

This approach has been considered theoretically for a few specific realizations of the cooling system: a Cooperpair box [19], the superconducting single-electron transistor [4,20,21], quantum dots [22], and ions [23]. However, for experimental simplicity one often prefers to get rid of the auxiliary quantum system mediating between cantilever and radiation field. This is achieved by coupling directly to the field of an optical cavity via radiation pressure. A number of recent experiments [24-31] have produced very promising results on self-cooling in such systems, using both photothermal and radiation pressure forces. In this Letter, we present a fully quantum-mechanical description, providing the basic theory for future ground-state cooling experiments. Cooling rates and steady-state tem- peratures for such systems (and related ones like a driven $L C$ circuit [32] ) have been derived only (semi-)classically so far. Specifically, Braginsky and Vyatchanin [33] considered the optomechanical damping within a semiclassical theory. Here, we use the quantum noise approach to find simple and transparent but fully quantum-mechanical expressions, starting directly from the force fluctuation spectrum. Our results are valid both for the good-cavity regime (resolved mechanical sidebands) and the bad-cavity regime (unresolved sidebands). Earlier (semi-)classical calculations $[24,32,33]$ are reproduced in the appropriate limits. We show, in particular, that it should be possible to cool the cantilever to its quantum-mechanical ground state by choosing the cantilever resonance frequency much larger than the cavity ringdown rate, a regime that has not been considered so far. Ground-state cooling is required for the experiments mentioned above and may permit studies of the optomechanical instability [28,34-36] in the quantum regime. Moreover, we present an exact solution of the linearized coupled equations of motion to account for the "strong cooling" limit, where the cooling rate exceeds the cavity ringdown rate.

Consider a mechanical degree of freedom $\hat{x}$ coupled parametrically with strength $A$ to the cavity oscillator

$$
\begin{aligned}
\hat{H}= & \hbar\left(\omega_{R}-A \hat{x}\right)\left[\hat{a}^{\dagger} \hat{a}-\left\langle\hat{a}^{\dagger} \hat{a}\right\rangle\right]+\hat{H}_{M}+\hat{H}_{\text {drive }} \\
& +\hat{H}_{\kappa}+\hat{H}_{\Gamma},
\end{aligned}
$$

where $\omega_{R}$ is the cavity resonance frequency at the equilibrium cantilever position $x=0$ in the presence of the mean radiation pressure, $\hat{H}_{M}=\hbar \omega_{M} \hat{c}^{\dagger} \hat{c}$ is the mechanical oscillator, $\hat{H}_{\text {drive }}$ is the optical drive, $\hat{H}_{\kappa}$ is the cavity damping, and $\hat{H}_{\Gamma}$ is the mechanical damping. For a cavity of length $L$, the coupling constant is $A=+\omega_{R} / L$.

Opening the port used to supply the classical drive to the cavity also admits vacuum noise. Splitting the cavity field 
into classical and quantum parts $\hat{a}(t)=e^{-i \omega_{L} t}[\bar{a}+\hat{d}(t)]$ yields the photon number autocorrelation function (in the absence of coupling to the cantilever)

$$
\begin{aligned}
\tilde{S}_{n n}(t) & =\left\langle\hat{a}^{\dagger}(t) \hat{a}(t) \hat{a}^{\dagger}(0) \hat{a}(0)\right\rangle-\left\langle\hat{a}^{\dagger}(t) \hat{a}(t)\right\rangle^{2} \\
& =\bar{n} e^{i \Delta t-(\kappa / 2)|t|},
\end{aligned}
$$

where $\Delta=\omega_{L}-\omega_{R}$ is the laser-cavity detuning and $\bar{n}$ is the mean photon number.

The power spectrum of the noise is

$$
S_{n n}(\omega)=\int_{-\infty}^{+\infty} d t e^{i \omega t} \tilde{S}_{n n}(t)=\bar{n} \frac{\kappa}{(\omega+\Delta)^{2}+(\kappa / 2)^{2}} .
$$

The symmetrized spectrum was used in [33] to estimate the effects of the shot noise (assumed to dominate over technical laser noise). Note, however, that it is the asymmetry in the noise which leads to cooling or heating. The noise of the radiation pressure force $\hat{F}=\hbar A \hat{n}$ at positive frequency, $S_{F F}\left(+\omega_{M}\right)$, corresponds to the ability of the cavity to absorb a quantum of energy from the cantilever, while noise at negative frequency $S_{F F}\left(-\omega_{M}\right)$ corresponds to emission of energy into the cantilever. The net optical damping rate of the cantilever from Fermi's golden rule is

$$
\Gamma_{\mathrm{opt}}=\frac{1}{\hbar^{2}}\left[S_{F F}\left(\omega_{M}\right)-S_{F F}\left(-\omega_{M}\right)\right] x_{\mathrm{ZPF}}^{2},
$$

where $x_{\mathrm{ZPF}}$ is the cantilever zero-point fluctuation and $S_{F F}=\hbar^{2} A^{2} S_{n n}$. This formula requires both $\Gamma_{\text {opt }}+$ $\Gamma_{M} \ll \kappa$ and a large mechanical quality factor, $Q_{M}=$ $\omega_{M} / \Gamma_{M} \gg 1$.

Essentially, the interaction with $\hat{x}$ (three wave mixing) tries to Raman scatter the drive photons into the high density of states at the cavity resonance. For negative detuning $\Delta<0$, this is uphill in energy [see Eq. (3) and Fig. 1(a)], and $\hat{x}$ is cooled. A somewhat similar mechanism
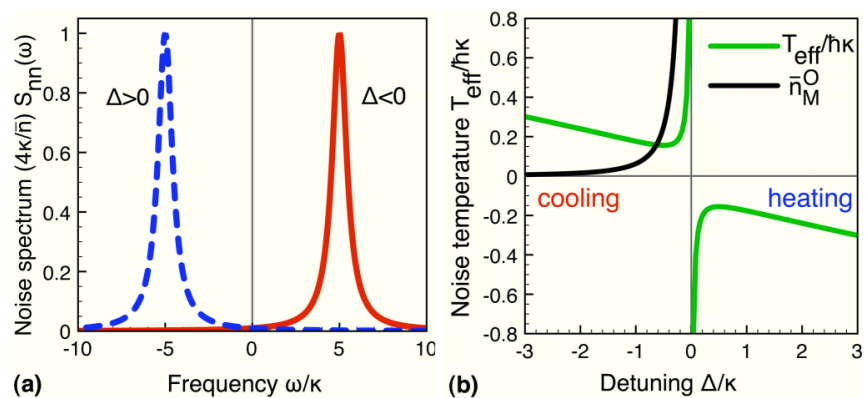

FIG. 1 (color online). (a) Noise spectrum of the photon number in a driven cavity as a function of frequency when the drive is detuned from the resonance by $\Delta=+5 \kappa$ (leading to heating: dashed line) and $\Delta=-5 \kappa$ (cooling: solid line). (b) Effective noise temperature $T_{\text {eff }}$ as a function of the detuning, see Eq. (5), for a cantilever frequency matching the detuning: $\omega_{M}=|\Delta|$. The noise temperature is positive on the left side of the resonance, where optomechanical cooling produces a minimum reachable cantilever phonon number $\bar{n}_{M}^{O}$ (black line) corresponding to $T_{\text {eff }}$, see Eqs. (5) and (7). involving an oscillator with nonlinear damping was considered by Dykman [37].

The nonequilibrium noise can be assigned a unique effective temperature $T_{\text {eff }}$, provided the cantilever is perfectly harmonic with weak optical and mechanical damping, $\Gamma_{M}+\Gamma_{\text {opt }} \ll \kappa$, so that it responds only at frequencies $\pm \omega_{M}$. Thus, without mechanical damping the mean steady-state number of cantilever phonons $\bar{n}_{M}^{O}$ is given by the detailed balance expression (for $\Delta<0$ )

$$
\frac{\bar{n}_{M}^{O}+1}{\bar{n}_{M}^{O}}=\frac{S_{F F}\left(+\omega_{M}\right)}{S_{F F}\left(-\omega_{M}\right)}=\exp \left(\frac{\hbar \omega_{M}}{T_{\text {eff }}}\right) .
$$

Cooling (heating) is indicated by $T_{\text {eff }}>0(<0)$ (see Fig. 1). From Eq. (3) we obtain

$$
\bar{n}_{M}^{O}=-\frac{\left(\omega_{M}+\Delta\right)^{2}+(\kappa / 2)^{2}}{4 \omega_{M} \Delta} .
$$

For the special case of detuning $\Delta=-\omega_{M}$ (which is optimal for $\left.\omega_{M} \gg \kappa\right)$, we have the simple limit

$$
\bar{n}_{M}^{O}=\left(\frac{\kappa}{4 \omega_{M}}\right)^{2},
$$

which shows that for $\omega_{M} \gtrsim \kappa$, the ground state can be approached (provided $\Gamma_{\mathrm{opt}} \gg \Gamma_{M}$ ). For the case $\Delta=$ $-\omega_{M}$, we obtain from Eq. (4):

$$
\Gamma_{\mathrm{opt}}=4\left(\frac{x_{\mathrm{ZPF}}}{L}\right)^{2} \frac{\omega_{R}^{2} \bar{n}}{\kappa} \frac{1}{1+\left(\frac{\kappa}{4 \omega_{M}}\right)^{2}} .
$$

After including the mechanical damping $\Gamma_{M}$, a rate equation yields the full expression for the mean steady-state phonon number (Fig. 2),

$$
\bar{n}_{M}=\frac{\Gamma_{\mathrm{opt}} \bar{n}_{M}^{O}+\Gamma_{M} \bar{n}_{M}^{T}}{\Gamma_{\mathrm{opt}}+\Gamma_{M}},
$$

where $\bar{n}_{M}^{T}$ is the equilibrium mechanical mode occupation number determined by the mechanical bath temperature. For $\bar{n}_{M}^{T} \gg 1$, Eq. (9) reduces to the classical expression. Note that ground-state cooling is possible only for initial phonon numbers $\bar{n}_{M}^{T} \ll Q_{M}$.

We emphasize once more that a large detuning $|\Delta|=$ $\omega_{M} \gg \kappa$ offers the advantage of an arbitrarily small minimum phonon number (7). The only price to pay is to increase the input intensity, in order to keep constant the number of photons inside the cavity, $\bar{n}=\bar{n}_{\max } /[1+$ $\left.(2 \Delta / \kappa)^{2}\right]$, where $\bar{n}_{\max }$ is the photon number at resonance, proportional to the input power. A large detuning increases the cooling efficiency in terms of the circulating power, thus limiting the heating of the cantilever by residual absorption of photons.

The optomechanical frequency shift (see, e.g., [30,33]) can also be derived from the force spectrum:

$$
\delta \omega_{M}=\frac{x_{\mathrm{ZPF}}^{2}}{\hbar^{2}} \int \frac{d \omega}{2 \pi} S_{F F}(\omega)\left[\frac{1}{\omega_{M}-\omega}-\frac{1}{\omega_{M}+\omega}\right] .
$$




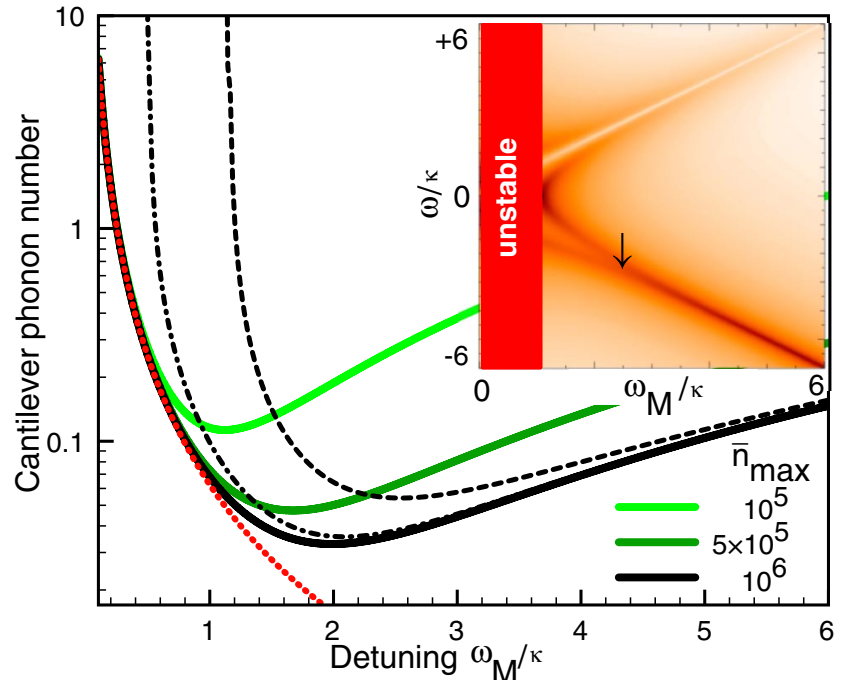

FIG. 2 (color online). The steady-state phonon number $\bar{n}_{M}$ obtained through cavity sideband cooling as a function of detuning, with $\Delta=-\omega_{M}$. Thick lines represent the weakcoupling quantum noise result, Eq. (9), for three different laser powers (expressed via constant $\bar{n}_{\max }$, so the actual photon number $\bar{n}$ increases towards smaller $\omega_{M}$ ). The bath temperature corresponds to $\bar{n}_{M}^{T}=100$ phonons, and the optomechanical coupling has been fixed to yield a realistic ratio $\Gamma_{\text {opt }}(\bar{n}=$ $\left.1, \omega_{M} \rightarrow \infty\right) / \Gamma_{M}=0.1$. The dotted red line shows $\bar{n}_{M}^{O}=$ $\left[\kappa /\left(4 \omega_{M}\right)\right]^{2}$ from Eq. (7). The dashed (dash-dotted) lines show the results of the exact input-output equations, for $\Gamma_{M} / \kappa=$ $10^{-4}\left(10^{-5}\right)$ and $\bar{n}_{\max }=10^{6}$. They were obtained by integrating the exact spectrum $S_{c c}(\omega)$, Eq. (13), which is shown in the inset as a density plot, for $\Gamma_{M} / \kappa=10^{-4}$ (arrow indicates splitting of resonance; "unstable" regime does not permit $\left.\Delta=-\omega_{M}\right)$. The weak-coupling limit is recovered for $\Gamma_{M} / \kappa \rightarrow 0$.

Equations (4), (5), (9), and (10) are valid for arbitrary spectra $S_{F F}$. These might result from the contribution of more than one mode, or relate to a different setup, e.g., a driven electrical circuit [32]. A related discussion [20] has considered cooling by a single-electron transistor.

We now compare with commonly employed simpler models [24,26,32], where one postulates the light intensity to relax in an exponential fashion, $d n / d t=\gamma(\bar{n}(x)-n)$, with $\bar{n}(x)$ denoting the equilibrium photon number as a function of position. After linearization, one obtains an effective optomechanical damping rate $[24,26,32] \Gamma_{\text {opt }}^{\prime}=$ $\left(\hbar A^{2} / m \gamma\right)\left[1+\left(\omega_{M} / \gamma\right)^{2}\right]^{-1} \frac{\partial \bar{n}}{\partial \Delta}$. In general, for a fixed $\gamma \propto$ $\kappa$, this differs from the correct result (4), and it predicts a maximum $\Gamma_{\mathrm{opt}}$ for $\gamma \sim \omega_{M}$. For radiation pressure cooling, one has to employ a detuning-dependent decay rate, $\gamma \equiv$ $\left[\left(\frac{\kappa}{2}\right)^{2}+\Delta^{2}\right] / \kappa$, to recover Eq. (4) in the limit $\omega_{M} \ll \kappa$. For bolometric forces, where the time lag is due to a finite heat relaxation rate $\gamma \ll \kappa$, our quantum theory does not apply due to the dissipative nature of the force, while $\Gamma_{\mathrm{opt}}^{\prime}$ is valid in the semiclassical limit.

The quantum result (4) for $\Gamma_{\mathrm{opt}}$ can be reproduced by linearizing the equation for the complex light amplitude $a$ $[29,33], d a / d t=i(\Delta+A x) a-\frac{\kappa}{2}(a-\bar{a})$. This still does not yield the correct steady-state phonon number (9), unless zero-point fluctuations are included.

To go beyond weak coupling, we now derive an exact solution of the linearized Heisenberg equations of motion for $\hat{d}$ and $\hat{c}$, using the input-output formalism [38] (see also [27]):

$$
\begin{gathered}
\dot{\hat{d}}=i \Delta \hat{d}-\frac{\kappa}{2} \hat{d}-\sqrt{\kappa} \hat{d}_{\mathrm{in}}+i \alpha\left(\hat{c}+\hat{c}^{\dagger}\right) \\
\dot{\hat{c}}=-i \omega_{M} \hat{c}-\frac{\Gamma_{M}}{2} \hat{c}-\sqrt{\Gamma_{M}} \hat{c}_{\mathrm{in}}+i\left(\alpha^{*} \hat{d}+\alpha \hat{d}^{\dagger}\right) .
\end{gathered}
$$

Here the effective light amplitude has been expressed in terms of a frequency, $\alpha=\bar{a}\left(\omega_{R} x_{\mathrm{ZPF}} / L\right)$ with $|\bar{a}|^{2}=\bar{n}$. The solution yields the cantilever spectrum $S_{c c}(\omega)=$ $\int d t e^{i \omega t}\left\langle\hat{c}^{\dagger}(t) \hat{c}\right\rangle$ :

$$
S_{c c}(\omega)=\frac{\Gamma_{M} \sigma_{\mathrm{th}}(\omega)+\frac{|\alpha|^{2}}{\kappa} \sigma_{\mathrm{opt}}(\omega)}{|\mathcal{N}(\omega)|^{2}}
$$

where

$$
\begin{aligned}
\sigma_{\mathrm{th}}(\omega) & =\left(\bar{n}_{M}^{T}+1\right)|\Sigma(\omega)|^{2}+\bar{n}_{M}^{T}\left|\chi_{M}^{-1}(\omega)+i \Sigma(\omega)\right|^{2}, \\
\sigma_{\mathrm{opt}}(\omega) & =\kappa^{2}\left|\chi_{R}(\omega)\right|^{2}\left|\chi_{M}^{-1}(\omega)\right|^{2}, \\
\mathcal{N}(\omega) & =\chi_{M}^{-1}(\omega) \chi_{M}^{-1 *}(-\omega)+2 \omega_{M} \Sigma(\omega) .
\end{aligned}
$$

We introduced the response functions of mirror and optical resonator, $\chi_{M}(\omega)=\left[\frac{\Gamma_{M}}{2}-i\left(\omega-\omega_{M}\right)\right]^{-1}$ and $\chi_{R}(\omega)=$ $\left[\frac{\kappa}{2}-i(\omega+\Delta)\right]^{-1}$, and we defined the optomechanical "self-energy" $\Sigma(\omega)=-i|\alpha|^{2}\left[\chi_{R}(\omega)-\chi_{R}^{*}(-\omega)\right]$.

The quantum noise results given previously are valid in the weak-coupling limit $\Gamma_{M}, \Gamma_{\mathrm{opt}} \ll \kappa$. Then, the optomechanical damping and the "optical spring" frequency shift can be read off the self-energy as $\operatorname{Im} \Sigma\left(\omega_{M}\right)=-\Gamma_{\text {opt }} / 2$ and $\operatorname{Re} \Sigma\left(\omega_{M}\right)=\delta \omega_{M}$, coinciding with the expressions given above [Eqs. (4) and (10)]. The steady-state average phonon number $\bar{n}_{M}=\int \frac{d \omega}{2 \pi} S_{c c}(\omega)$ reproduces Eq. (9).

The optical output spectrum (of $\hat{d}_{\text {out }}=\hat{d}_{\text {in }}+\sqrt{\kappa} \hat{d}$ ) displays a Stokes peak at $\omega=-\omega_{M}$ and an anti-Stokes peak at $\omega=+\omega_{M}$, with weights given by $\Gamma_{\text {opt }}\left(\bar{n}_{M}+1\right) \bar{n}_{M}^{O}$ and $\Gamma_{\text {opt }} \bar{n}_{M}\left(\bar{n}_{M}^{O}+1\right)$, respectively, which are the rates of processes leading to heating (cooling) of the cantilever by redshifting (blueshifting) a reflected photon. The ratio of the peak intensities thus provides a measure of the phonon number $\bar{n}_{M}$, and the anti-Stokes peak vanishes upon reaching the mechanical ground state.

The exact solution allows us also to discuss the regime of strong cooling. In the limit $\Gamma_{\mathrm{opt}} \approx 4|\alpha|^{2} / \kappa \gg \Gamma_{M}$, it adds a term $\bar{n}_{M}^{T} \Gamma_{M} / \kappa+2 \bar{n}_{M}^{O} \Gamma_{\text {opt }} / \kappa$ to Eq. (9) for $\bar{n}_{M}$ (assuming $\kappa, \alpha \ll \omega_{M}$, and $\Gamma_{M} \ll \kappa$ ). Then a minimal phonon number $\bar{n}_{M}^{\min } \geq \bar{n}_{M}^{T} \Gamma_{M} / \kappa$ is found as a function of $\Gamma_{\text {opt }}$ at $\left(\Gamma_{\text {opt }} / \kappa\right)^{2}=\left(\Gamma_{M} / \kappa\right)\left(\bar{n}_{M}^{T} / \bar{n}_{M}^{O}\right) / 2$. For $\Gamma_{\text {opt }} / \kappa>$ $1 / 2$, the mirror resonance peak splits into a pair of peaks at $-\omega_{M} \pm \alpha$, getting hybridized with the driven cavity mode (see arrow in inset of Fig. 2). At even larger photon number or smaller $\omega_{M}$, when $\alpha \approx \omega_{M} / 2$, the static bistability [39] 
precludes reaching the desired detuning $\Delta=-\omega_{M}$ (see inset of Fig. 2). Thus, the far-detuned regime $\omega_{M} \gg \kappa$ has the additional strong advantage of avoiding the bistability, which already interferes with cooling in some current schemes [29].

Recent experiments [26,28-31] combine all the necessary ingredients and some of them $[28,29,31]$ have already demonstrated $\omega_{M} / \kappa \sim 1$, the threshold of the good-cavity regime. As an example of what might be possible in the near future, we mention estimates for two realistic setups: a microtoroidal resonator [29] with $\omega_{M} / 2 \pi=60 \mathrm{MHz}$, $\kappa / 2 \pi=50 \mathrm{MHz}$, and $Q_{M}=10^{4}$, and a mechanical membrane between mirrors [31] with $\omega_{M} / 2 \pi=100 \mathrm{kHz}$, $\kappa / 2 \pi=8 \mathrm{kHz}$, and $Q_{M}=4 \cdot 10^{6}$. Both of these could be cooled down to $\bar{n}_{M}<1$ after precooling to $T=$ $300 \mathrm{mK}$. This could be achieved using $P_{\text {in }} \sim 5 \mathrm{~mW}$ (or $P_{\text {in }} \sim 1 \mathrm{nW}$, respectively), while remaining in the weakcoupling regime $\Gamma_{\text {opt }} \ll \kappa \sim \omega_{M}$.

We have obtained the full quantum theory of cavityassisted sideband cooling of a cantilever, based on the quantum noise approach applied to the fluctuations of the radiation pressure. Only in the previously unexplored regime of a detuning much larger than the cavity linewidth can the cantilever be cooled to arbitrarily small phonon numbers. The theory analyzed here may form the basis for future experiments that venture into the quantum regime of mechanical motion.

We thank M. Dykman, I. Wilson-Rae, and T. Kippenberg for comments, and particularly J. Harris for useful contributions. This work was supported in part by the NSF under Grants No. ITR-0325580, No. DMR0342157, and No. DMR-0603369, by the NSERC, and by the SFB 631 and NIM of the DFG.

Note added.-Essentially similar conclusions were reached recently in [40].

[1] A. Erbe, C. Weiss, W. Zwerger, and R. H. Blick, Phys. Rev. Lett. 87, 096106 (2001).

[2] X. M. H. Huang, C. A. Zorman, M. Mehregany, and M. L. Roukes, Nature (London) 421, 496 (2003).

[3] R. G. Knobel and A.N. Cleland, Nature (London) 424, 291 (2003).

[4] A. Naik et al., Nature (London) 443, 193 (2006).

[5] M.P. Blencowe and M.N. Wybourne, Physica (Amsterdam) 280B, 555 (2000).

[6] S. Mancini, V. I. Manko, and P. Tombesi, Phys. Rev. A 55, 3042 (1997).

[7] S. Bose, K. Jacobs, and P. L. Knight, Phys. Rev. A 59, 3204 (1999).

[8] W. Marshall, C. Simon, R. Penrose, and D. Bouwmeester, Phys. Rev. Lett. 91, 130401 (2003).

[9] A. D. Armour, M. P. Blencowe, and K. C. Schwab, Phys. Rev. Lett. 88, 148301 (2002).

[10] J. Zhang, K. Peng, and S. L. Braunstein, Phys. Rev. A 68 , 013808 (2003).
[11] M. Pinard, A. Dantan, D. Vitali, O. Arcizet, T. Briant, and A. Heidmann, Europhys. Lett. 72, 747 (2005).

[12] V. B. Braginsky and F. Y. Khalili, Quantum Measurement (Cambridge University Press, Cambridge, England, 1992).

[13] M. D. LaHaye, O. Buu, B. Camarota, and K. C. Schwab, Science 304, 74 (2004).

[14] D. J. Wineland and W. M. Itano, Phys. Rev. A 20, 1521 (1979).

[15] J.M.W. Milatz and J.J. van Zolingen, Physica (Amsterdam) 19, 181 (1953).

[16] P. F. Cohadon, A. Heidmann, and M. Pinard, Phys. Rev. Lett. 83, 3174 (1999).

[17] D. Kleckner and D. Bouwmeester, Nature (London) 444, 75 (2006).

[18] J. M. Courty, A. Heidmann, and M. Pinard, Eur. Phys. J. D 17, 399 (2001).

[19] I. Martin, A. Shnirman, L. Tian, and P. Zoller, Phys. Rev. B 69, 125339 (2004).

[20] A. A. Clerk and S. Bennett, New J. Phys. 7, 238 (2005).

[21] M.P. Blencowe, J. Imbers, and A.D. Armour, New J. Phys. 7, 236 (2005).

[22] I. Wilson-Rae, P. Zoller, and A. Imamoglu, Phys. Rev. Lett. 92, 075507 (2004).

[23] L. Tian and P. Zoller, Phys. Rev. Lett. 93, 266403 (2004).

[24] C. Höhberger-Metzger and K. Karrai, Nature (London) 432, 1002 (2004); note that in Eq. (7), the frequency ratio should be dropped [K. Karrai (private communication)].

[25] J. G. E. Harris, B. M. Zwickl, and A. M. Jayich, Rev. Sci. Instrum. 78, 013107 (2007).

[26] S. Gigan et al., Nature (London) 444, 67 (2006).

[27] M. Paternostro, S. Gigan, M.S. Kim, F. Blaser, H. R. Böhm, and M. Aspelmeyer, New J. Phys. 8, 107 (2006).

[28] O. Arcizet, P. F. Cohadon, T. Briant, M. Pinard, and A. Heidmann, Nature (London) 444, 71 (2006).

[29] A. Schliesser, P. Del'Haye, N. Nooshi, K. J. Vahala, and T. J. Kippenberg, Phys. Rev. Lett. 97, 243905 (2006).

[30] T. Corbitt, Y. Chen, E. Innerhofer, H. Muller-Ebhardt, D. Ottaway, H. Rehbein, D. Sigg, S. Whitcomb, C. Wipf, and N. Mavalvala, Phys. Rev. Lett. 98, 150802 (2007).

[31] J.D. Thompson, B. M. Zwickl, A.M. Jayich, F. Marquardt, S.M. Girvin, and J.G.E. Harris, arXiv:0707.1724.

[32] D. J. Wineland et al., arXiv:quant-ph/0606180.

[33] V. B. Braginsky and S. P. Vyatchanin, Phys. Lett. A 293, 228 (2002).

[34] T. Carmon, H. Rokhsari, L. Yang, T. J. Kippenberg, and K. J. Vahala, Phys. Rev. Lett. 94, 223902 (2005).

[35] F. Marquardt, J. G. E. Harris, and S. M. Girvin, Phys. Rev. Lett. 96, 103901 (2006).

[36] T. Corbitt et al., Phys. Rev. A 74, 021802(R) (2006).

[37] M. Dykman, Sov. Phys. Solid State 20, 1306 (1978).

[38] D.F. Walls and G. J. Milburn, Quantumoptics (Springer, New York, 1995).

[39] A. Dorsel, J. D. McCullen, P. Meystre, E. Vignes, and H. Walther, Phys. Rev. Lett. 51, 1550 (1983).

[40] I. Wilson-Rae, N. Nooshi, W. Zwerger, and T.J. Kippenberg, preceding Letter, Phys. Rev. Lett. 99, 093901 (2007). 